\title{
Sphingosine-1-phosphate metabolism: can its enigmatic lyase promote the autophagy of fibrosis?
}

\author{
Amanda L Tatler, Gisli Jenkins
}

Idiopathic pulmonary fibrosis (IPF) is a devastating disease with a poorly understood pathogenesis and a rising incidence. ${ }^{1}$ Recently, an important role for bioactive lipids such as sphingosine-1phosphate (S1P) and lysophosphatidic acid (LPA), which have a diverse range of cellular functions including proliferation, cell death, adhesion and migration, has emerged. Both S1P and LPA are increased within the bronchoalveolar lavage fluid of patients with IPF $^{2} 3$ and levels of sphingosine kinase1/2 (SPK1/2), which catalyses the synthesis of S1P from sphingosine, correlate negatively with survival in patients with IPF, ${ }^{4}$ highlighting an important role in disease.

Studies have shown that there is crosstalk between bioactive lipids such as S1P and LPA and the potently pro-fibrotic cytokine transforming growth factor $\beta$ (TGF- $\beta$ ), which is known to be fundamental to IPF pathogenesis. ${ }^{5}$ Both S1P and LPA may promote lung fibrosis via activation of TGF- $\beta$ in multiple cell types. ${ }^{6-8}$ Activation of TGF- $\beta$ by S1P and LPA occurs via signalling through their respective G-protein coupled receptors. In vivo animal studies have shown that loss of such receptors, including LPAR1, LPAR2 or S1P3 receptors, can ameliorate bleomycin-induced lung fibrosis in rodents. ${ }^{3} 910$ Furthermore, expression of SPK $1 / 2$ is TGF- $\beta$ dependent, ${ }^{4}$ highlighting a positive feedback loop that could perpetuate fibrogenesis.

In addition to bioactive lipids promoting TGF- $\beta$ activation and its downstream functional effects, there is emerging evidence that both the bioactive lipids S1P and LPA, as well as TGF- $\beta$ itself, inhibit autophagy. Autophagy is a cellular process that degrades cytoplasmic substrates and organelles to obtain energy and metabolic building blocks. It is necessary for the

Centre for Respiratory Research, University of Nottingham, Nottingham City Hospital, Nottingham, UK

Correspondence to Dr Gisli Jenkins, Centre for Respiratory Research, University of Nottingham, Nottingham City Hospital, Clinical Sciences Building Nottingham NG51PB, UK;

gisli.jenkins@nottingham.ac.uk homeostasis of organelle integrity, clearance of aggregated and misfolded proteins, and for tissue remodelling during development and infection resistance. Lung tissue obtained from patients with IPF shows reduced autophagic processes through the actions of TGF- $\beta$ on mechanistic target of rapamycin (mTOR), ${ }^{11}$ which is a central negative regulator of autophagy. Supporting a role for mTOR and autophagy in fibrogenesis, mTOR levels are increased in an in vivo model of systemic sclerosis. ${ }^{12}$ Likewise, S1P can reduce autophagy through an mTOR-dependent pathway. ${ }^{13}$ Importantly, inhibiting $\mathrm{mTOR}$, and therefore increasing autophagy, is sufficient to reduce bleomycin-induced fibrosis in the lung. ${ }^{14}$

In this issue of Thorax, Huang and colleagues show that overexpression of S1P lyase (S1PL), which degrades S1P and therefore inhibits S1P signalling, can increase markers of autophagy in lung fibroblasts. Furthermore, using $\mathrm{S}_{\mathrm{PL}} \mathrm{PL}^{+/}$ mice they demonstrate increased pulmonary S1P levels, reduced markers of autophagy and an enhanced fibrotic response to bleomycin further supporting a role for S1P and autophagy in the development of tissue fibrosis, and the authors suggest that S1PL is, therefore, an endogenous suppressor of pulmonary fibrosis. ${ }^{15}$ Intriguingly, however, the authors also demonstrate both TGF- $\beta$ and bleomycin lead to increased expression of S1PL, and furthermore, that levels of S1PL are increased in lung tissue and lung fibroblasts obtained from patients with IPF. This would suggest that if S1PL is an endogenous suppressor of pulmonary fibrosis, in many cases the response is either insufficient or futile in patients with IPF. Fundamentally, the authors show that increased levels of S1PL messenger RNA in peripheral blood mononuclear cells were associated with higher diffusing capacity of lungs for carbon monoxide (DLCO) and improved survival in patients with IPF, ${ }^{15}$ suggesting that augmenting autophagy in pulmonary fibrosis may be an attractive therapeutic response.

It is likely that modulating the S1P pathway through increasing S1PL is going to be challenging, if possible at all, and would require S1PL overexpression through gene therapy as we are not aware of any molecules that specifically augment S1PL in vivo. S1PL inhibitors have recently been developed for the treatment of multiple sclerosis ${ }^{16}$ and understanding their effects in models of fibrosis will be important in light of the study by Huang et al. ${ }^{15}$ Therefore, investigating molecules that target the downstream effects of the S1P pathway may hold more promise.

Nintedanib, already approved for clinical use in IPF, has recently been shown to exhibit antifibrotic effects partly through increased autophagy. ${ }^{17}$ Furthermore, rapamycin and metformin, both of which are already used clinically in non-fibrotic diseases, have recently been shown to impact the mTOR pathway and increase autophagy, ${ }^{18}$ suggesting that they may have potential for repurposing in IPF. Rapamycin, which targets the mTOR pathway, is widely used as an antirejection therapy following kidney transplant. It has, however, shown efficacy as an antifibrotic drug in both the lung and skin in in vivo models. $^{12} 19$ Targeting autophagy with rapalogues has been tried in patients with $\mathrm{IPF}^{20}$ and although the results of the everolimus study did not support its use, everolimus inhibits only the mTORC1 complex and it is possible that dual mTOR complex inhibition is required. Metformin may have even more potential as an adjunctive therapy in fibrotic disease, it is a widely prescribed antidiabetic therapy with a therapeutic and safety profile that is well known and it has demonstrable antifibrotic effects in various organ systems. ${ }^{21} 22$ In the lung, metformin is effective at reducing fibrotic airway remodelling in response to both bleomycin and allergen challenge. ${ }^{23}$

Over and above the primary mechanistic insights into S1PL in the development of IPF, the paper by Huang et $a l^{15}$ highlights some important issues in our quest to understand, and better treat, IPF. First, the increased expression of S1PL in IPF does not indicate it is involved in the pathogenesis of disease and emphasises the importance of functional experiments to complement observational studies. Second, they identify bioactive lipidmediated autophagy as an important pathway in the pathogenesis of IPF, and finally, this study demonstrates that understanding the basic mechanisms of disease may help identify currently available drugs that could hold promise if repurposed for the treatment of IPF.

Competing interests None declared. 
Provenance and peer review Commissioned; internally peer reviewed.

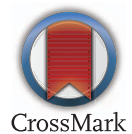

To cite Tatler AL, Jenkins G. Thorax 2015;70:11061107.

Published Online First 3 November 2015

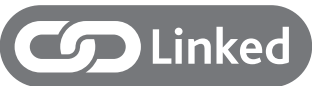

- http://dx.doi.org/10.1136/thoraxjnl-2014-206684

Thorax 2015;70:1106-1107.

doi:10.1136/thoraxinl-2015-207974

\section{REFERENCES}

1 Navaratnam V, Fleming KM, West J, et al. The rising incidence of idiopathic pulmonary fibrosis in the UK. Thorax 2011:66:462-7.

2 Milara J, Navarro R, Juan G, et al. Sphingosine-1phosphate is increased in patients with idiopathic pulmonary fibrosis and mediates epithelial to mesenchymal transition. Thorax 2012;67:147-56.

3 Tager AM, Lacamera P, Shea BS, et al. The lysophosphatidic acid receptor LPA1 links pulmonary fibrosis to lung injury by mediating fibroblast recruitment and vascular leak. Nat Med 2008:14:45-54.

4 Huang LS, Berdyshev E, Mathew B, et al. Targeting sphingosine kinase 1 attenuates bleomycin-induced pulmonary fibrosis. Faseb J 2013;27:1749-60.
5 Tatler AL, Jenkins G. TGF-beta activation and lung fibrosis. Proc Am Thorac Soc 2012:9:130-6.

6 Xu MY, Porte J, Knox AJ, et al. Lysophosphatidic Acid Induces \{alpha\}v\{beta\}6 Integrin-Mediated TGF-\{beta\} Activation via the LPA2 Receptor and the Small G Protein G\{alpha\}q. Am J Pathol 2009;74:1264-79.

7 Tatler $A L$, John $A E$, Jolly $L$, et al. Integrin alphavbeta5-mediated TGF-beta activation by airway smooth muscle cells in asthma. $J$ Immunol 2011;187:6094-107.

8 Giacomini MM, Travis MA, Kudo M, et al. Epithelial cells utilize cortical actin/myosin to activate latent TGF-beta through integrin alpha(v)beta (6)-dependent physical force. Exp Cell Res 2012;318:716-22.

9 Murakami K, Kohno M, Kadoya M, et al. Knock out of S1P3 receptor signaling attenuates inflammation and fibrosis in bleomycin-induced lung injury mice model. PLOS ONE 2014:9:e106792.

10 Huang LS, Fu P, Patel P, et al. Lysophosphatidic acid receptor-2 deficiency confers protection against bleomycin-induced lung injury and fibrosis in mice. Am J Respir Cell Mol Biol 2013;49:912-22.

11 Patel AS, Morse D, Choi AM. Regulation and functional significance of autophagy in respiratory cell biology and disease. Am J Respir Cell Mol Biol 2013:48:1-9.

12 Yoshizaki A, Yanaba K, Yoshizaki A, et al. Treatment with rapamycin prevents fibrosis in tight-skin and bleomycin-induced mouse models of systemic sclerosis. Arthritis Rheum 2010;62:2476-87.

13 Taniguchi M, Kitatani K, Kondo T, et al. Regulation of autophagy and its associated cell death by "sphingolipid rheostat": reciprocal role of ceramide and sphingosine 1-phosphate in the mammalian target of rapamycin pathway. J Biol Chem 2012;287:39898-910.
14 Gan X, Wang J, Wang C, et al. PRR5L degradation promotes mTORC2-mediated PKC-delta phosphorylation and cell migration downstream of Galpha12. Nat Cell Biol 2012;14:686-96.

15 Huang LS, Berdyshev EV, Tran JT, et al. Sphingosine-1-phosphate lyase is an endogenous suppressor of pulmonary fibrosis: role of S1P signalling and autophagy. Thorax 2015;70:1138-48.

16 Cosconati S, Novellino $E$. The first sphingosine 1-phosphate lyase inhibitors against multiple sclerosis: a successful drug discovery tale. J Med Chem 2014;57:5072-3.

17 Rangarajan S, Kurundkar A, Kurundkar D, et al. Novel Mechanisms for the Anti-Fibrotic Action of Nintedanib. Am J Respir Cell Mol Biol. Published Online First: 13 Jun 2015. doi:10.1165/rcmb.2014 $04450 C$

18 Kim YC, Guan KL. mTOR: a pharmacologic target for autophagy regulation. J Clin Invest 2015;125:25-32.

19 Simler NR, Howell DCJ, Marshall RP, et al. The rapamycin analogue SDZ RAD attenuates bleomycin-induced pulmonary fibrosis in rats. Eur Respir J 2002;19:1124-7.

20 Malouf MA, Hopkins P, Snell G, et al. An investigator-driven study of everolimus in surgical lung biopsy confirmed idiopathic pulmonary fibrosis. Respirology 2011;16:776-83.

21 Lu J, Shi J, Li M, et al. Activation of AMPK by metformin inhibits TGF-beta-induced collagen production in mouse renal fibroblasts. Life $\mathrm{SC}$ 2015;127:59-65.

22 Xiao $\mathrm{H}, \mathrm{Ma}$ X, Feng $\mathrm{W}$, et al. Metformin attenuates cardiac fibrosis by inhibiting the TGFbeta1-Smad3 signalling pathway. Cardiovasc Res 2010;87:504-13.

23 Park CS, Bang B-R, Kwon H-S, et al. Metformin reduces airway inflammation and remodeling via activation of AMP-activated protein kinase. Biochem Pharmacol 2012;84:1660-70. 